\title{
Novel mutation and severe respiratory failure in congenital disorders of glycosylation Type Ix
}

\author{
Betül Kılıç ${ }^{1 \oplus}$, Nejmiye Akkuş \\ Departments of ${ }^{1}$ Pediatric Neurology and ${ }^{2}$ Medical Genetics, University of Health Sciences, Kocaeli Derince Training and Research \\ Hospital, Kocaeli, Turkey.
}

\begin{abstract}
Congenital glycosylation disorders (CDG) are a group of rare hereditary metabolic diseases that result from abnormal protein and lipid glycosylation. Virtually all organ systems can be affected, and neurological involvement is particularly severe and disabling. More than 100 CDG types have been reported to date and those numbers are rapidly increasing. Each type is very rare, and the clinical characteristics of each subtype are difficult to determine. There are large numbers of biochemically unresolved cases defined as CDGIx. In this report, we present a 5-year-old boy who had dysmorphic features, hypotonia, developmental and mental delay, epileptic spasms, recurrent apnea and respiratory failure that led to the diagnosis of an unreported mutation of a rare form of CDG-Ix. This mutation in the STT3B gene affects the catalytic subunit of the oligosaccharyltransferase and the recipient substrate properties, which in part have the same functions in N-glycosylation. A novel homozygous mutation in the STT3B presence of c.38C $>\mathrm{G}$ that encodes p.S13W (p.Ser13Trp) was detected with next generation sequencing. The CDG clinical spectrum can be unusual, ranging from dysfunction of certain organs to severe multiple system disorders. Respiratory failure has rarely been reported in these cases. Increased types and numbers of patients constitute symptom variety. The identification of new genes and genotype-phenotype relationships may expand the family of CDG.
\end{abstract}

Key words: novel mutation, STT3B, congenital disorders of glycosylation Type Ix, respiratory failure.

Congenital disorders of glycosylation (CDG) comprise a group of inherited congenital metabolic disorders caused by protein or lipid glycosylation defects. To date, more than a hundred types of CDG have been defined although it is difficult to diagnose each subtype. ${ }^{1,2}$

In localization of intracellular molecular defects, CDG cases are categorized into two types: CDG I or II. ${ }^{4-7}$ Two types of defects in protein $\mathrm{N}$-glycosylation are related to a series of mixed pathways involving combining and processing proteins. These pathways occur in three cellular compartments: the Golgi apparatus, the endoplasmic reticulum and the cytosol. CDG-I

\footnotetext{
Betül Kılıç

betulklc82@gmail.com
}

Received 16th Januray 2019; revised 10th March 2019; accepted 19th March 2019 is caused by defects in the enzymes that provide the synthesis and transfer of the oligosaccharide in the endoplasmic reticulum (ER). Pathologies leading to CDG-II are associated with different mechanisms: enzymes involved in the regulation of the N-glycan chain in the Golgi apparatus, sugar carriers and intracellular proteins such as the COG complex. ${ }^{8}$

After the latest revision to the terminology, the CDG type is detected according to the affected gene name or protein name. ${ }^{4}$ However, the prevalence of this group of disorders is not exactly known. As long as the basic disorder remains unknown, diseases are called CDG-Ix. ${ }^{9}$

Patients with CDG-I present with mental and psychomotor delay, seizures, muscle hypotonia, neurological signs, ophthalmological anomalies, failure to thrive, blood coagulation defects, endocrine abnormalities, and volatile dysmorphic features. ${ }^{8}$ 
Approximately $15 \%$ of all CDG families are subtyped as CDG-Ix owing to unidentified glycosylation defects. ${ }^{11}$ These defects may include deficiencies in oligosaccharyltransferase (OST) activity or dolichol biosynthesis from mevalonate. These may include, for example, analysis of the dolichol biosynthetic pathway from mevalonate or measurement of OST activity.

In this article, we describe a 5-year-old patient with a rare unregistered respiratory failure who was diagnosed with CDG-Ix using new generation sequencing (NGS). We detected a homozygous mutation in $S T T 3 B$ and confirmed the presence of c.38C $>\mathrm{G}$ that encodes p.S13W (p.Ser13Trp) and were able to diagnose CDG-Ix. The patient had episodes of infantile spasm and showed symptoms of respiratory failure, which are very rarely reported in CDG Ix.

\section{Case Report}

A 5-year-old boy was the first-born child of healthy and consanguineous Turkish parents. Lower fetal movements and intrauterine growth retardation were reported during pregnancy. He was born at week 38 and weighed $2660 \mathrm{~g}$ (SD, $-3.25)$ with a length of $47 \mathrm{~cm}(\mathrm{SD},-4 \mathrm{SD})$, Apgar score of 10/10, and occipitofrontal circumference of $34 \mathrm{~cm}(\mathrm{SD},-2)$. At three months of age, few psychomotor acquisitions, dysmorphic features, generalized hyporeflexia, and hypotonia were noticed. The fundus oculi exhibited minor pale papillae. An electroretinogram recorded few responses to stimulation. Evoked responses in the auditory brainstem were normal. He developed epileptic spasms at the age of 1 and received benzodiazepine treatment.

The patient first visited our hospital due to an infection-related phase of respiratory insufficiency at five years of age. He demonstrated dysmorphic features including hirsutism, narrow forehead, short palpebral fissure, hypertelorism, antimongoloid slanting eyes, and long eyelashes. Clinical findings included axial hypotonia, body adiposity, muscular weakness, and progressive developmental delay. Moreover, recurrent apnea was observed, for which long-term oxygen supplementation with cannulation was initiated followed by invasive ventilation via tracheostomy.

Laboratory investigation results were normal for peripheral blood count, serum cholesterol, lactate, amino acids, albumin, alkaline phosphatase, prothrombin time and activated partial thromboplastin time, thyroid stimulating hormone, thyroxine, and thyroxinebinding globulin levels. A temporal increase in renal failure and serum transaminases was also noted. Metabolic screening (amino acids, plasma amino acid, urinary organic acids, ammonia, biotinidase, phytanic acid, lactate, vitamin $\mathrm{B} 12$, blood $\mathrm{pH}$, carnitine acyl carnitine levels, and long-chain fatty acids) revealed no abnormal findings.

Abdominal ultrasound and echocardiogram confirmed the absence of abnormalities in the liver and heart. However, renal ultrasound revealed bilateral, grade 1 pelviectasis. Electroencephalography examination revealed a burst suppression pattern. Brain magnetic resonance imaging showed a generalized reduction in myelination; moreover, the third and fourth ventricles were observed to be wide with gliotic changes around the lateral ventricle.

The high-performance liquid chromatography method was used for measurement of transferrin glycoforms in serum. Quantification of transferrin glycoforms was performed with selective absorbance at $\sim 214 \mathrm{~nm}$. All plasma transferrin levels (asialo-, monosialo-, disialo-, trisialo-, tetrasialo-, and pentasialotransferrin) were found to be normal (Fig. 1).

The patient's karyotype was 46, XY. In peripheral leukocytes, mitochondrial DNA and $X Y$ chromosome were in the normal order without any large deletion. Additionally, array comparative genomic hybridization revealed no abnormal findings. 


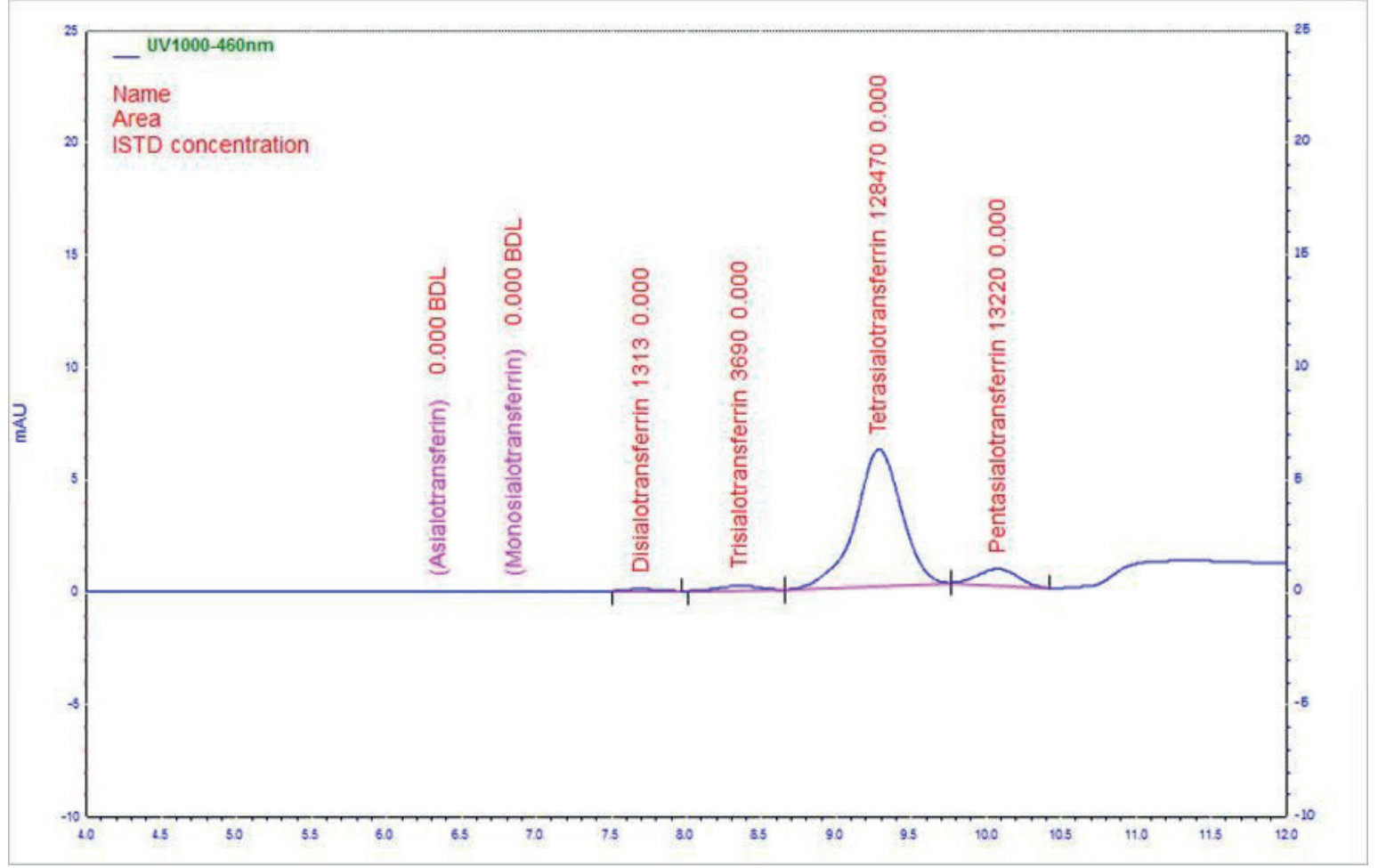

Fig. 1. Patient's plasma transferrin levels are in normal limits.

Genomic DNA was used to perform exome sequencing. Briefly, peripheral blood leukocytes were extracted and exomes were captured using TruSight One Panel (Illumina Inc., San Diego, CA, USA) that is capable of enriching a $12-\mathrm{Mb}$ region spanning 4813 genes. The NextSeq platform (Illumina Inc.) was used for sequencing. Burrow-Wheeler Aligner (version 0.7.12, MEM algorithm) was used for aligning sequence reads with those of the reference hg19 genome. ${ }^{3}$ Candidate variants were confirmed using custom-designed primers for Sanger sequencing.

Subsequently, NGS was used to diagnose CDG Ix by detecting a homozygous mutation in $S T T 3 B$ and with Sanger sequencing to confirm the presence of c. 38C>G that encodes p.S13W (p.Ser13Trp) (Fig. 2). Results of the genetic analysis revealed that the patient's parents were heterozygous for every mutated allele. Moreover, the patient was found to be homozygous for c.38C >G (NM_178862.1) that encodes protein p.Ser13Trp. This mutation is a new variation not found in the ClinVar database. However, the mutation found in SIFT, PolyPhen and MutationTaster databases is disease-specific. Written informed consent was obtained from the patient's parents for publication of the case.

\section{Discussion}

We report a patient with a formerly unlisted category of CDG arising from a mutation in $S T T 3 B$. STT3B is a $22.8-\mathrm{Mb}$ OST complex gene located on chromosome $3(9,771,508-$ $32,612,897)$.

OST catalyzes the transfer of the oligosaccharide from a lipid-linked oligosaccharide donor to the asparagine residue of glycosylation receptor sites or sequons in newly synthesized proteins. These enzymes are heteromeric complexes consisting of several membrane-related subunits, where STT3 is a catalytic center. ${ }^{12}$

The genome contains two different STT3 genes, STT3A and STT3B. Therefore, at least 


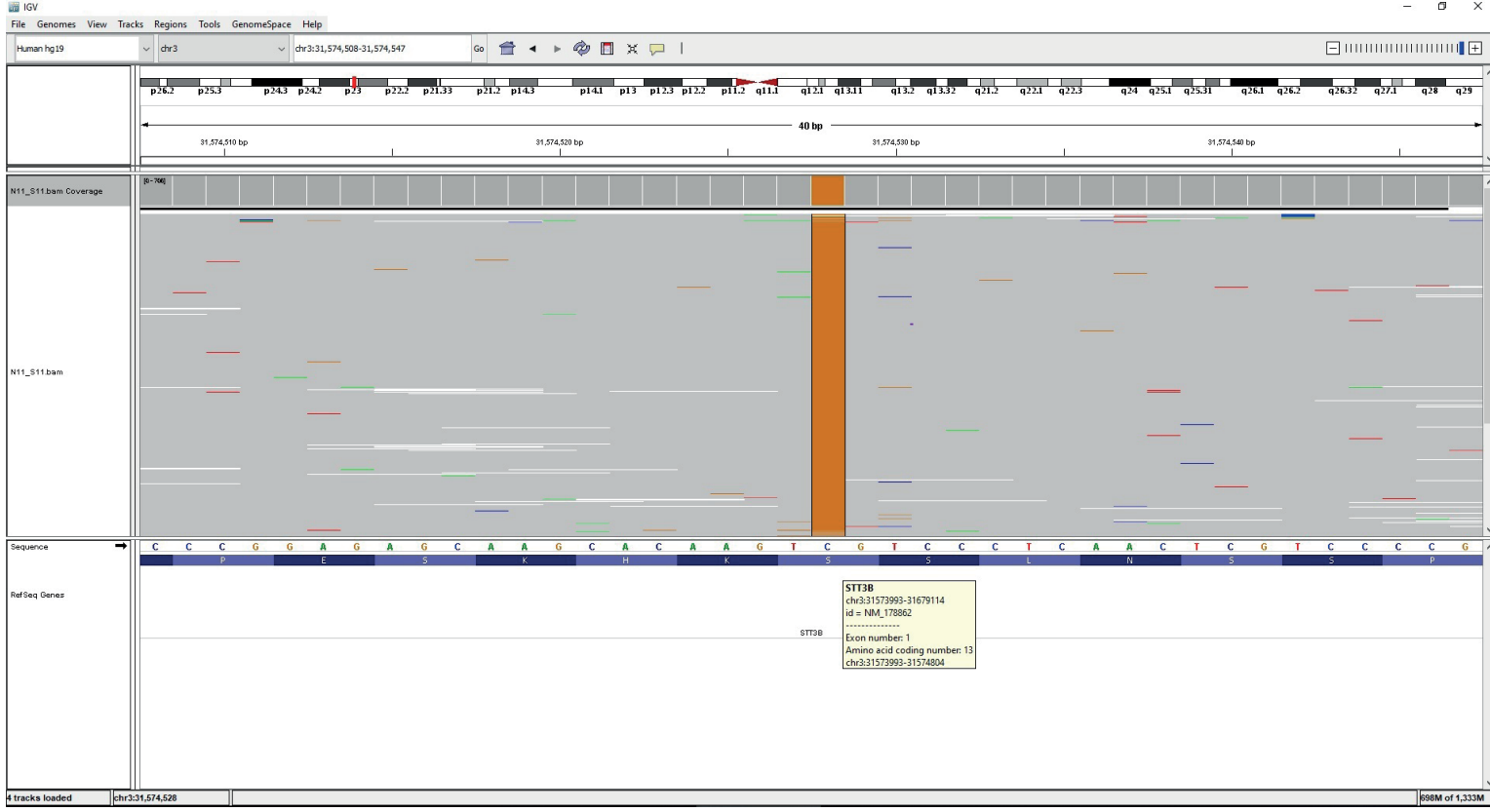

Fig. 2. Sanger sequencing showed the presence of c. 38C $>\mathrm{G}$ that encodes p.S13W (p.Ser13Trp).

two different OST isoforms are formed. The STT3A isoform primarily glycosylates substrate polypeptides cotranslationally, while the STT3B isoform provides cotranslational and post-translocational glycosylation of sequons that are skipped by the STT3A isoform. ${ }^{13}$

The oligosaccharides are transferred to N-linked glycoproteins with the STT3B isoform. It has also been reported that STT3Bdependent $\mathrm{N}$-glycosylation plays a role in clearing misfolded proteins due to ER-related degradation. ${ }^{14,15}$ The STT3B isoform is thought to have an important function in protein homeostasis in ER.

Measurement of transferrin glycoforms in serum is a laboratory method used to identify suspected CDG patients. However, since the protein is mainly glycosylated with the STT3A isoform of OST, potential CDG-STT3B cases cannot be determined by serum transferrin measurement. ${ }^{16,17}$

Almost all types of CDG manifest in infancy. As oligosaccharides in both glycolipids and glycoproteins have essential biological functions, defects in the synthesis of these molecules results in broad, multi-system clinical symptoms. ${ }^{18}$
These symptoms may include one or more of the following: failure to thrive, developmental delay, hepatopathy, hypotonia/neurological abnormalities, hypoglycemia, protein-losing enteropathy, eye abnormalities, abnormal immunological findings, skin abnormalities, and skeletal findings. ${ }^{19}$ For instance, an individual with STT3B mutation was reported to present with severe developmental delay, seizure disorder, microcephaly, failure to thrive and liver and genitourinary abnormalities. ${ }^{6,17}$

Most CDG types and multiple pathways manifest in an autosomal recessive manner. ALG13-, MGAT1-, SSR4-, and SLC35A2-CDG manifest in an X-linked manner. Homozygous mutations in genes located on chromosome 3 p23 result in STT3B-CDG (CDG-Ix). ${ }^{20}$

Our patient had significant congenital and developmental abnormalities. The homozygous mutation (c.38C> G) in STT3B causes a significant reduction in $S T T 3 B$ protein and hypoglycosylation of STT3B-specific substrates. In our case, the parents were heterozygous for every mutated allele and healthy. Accordingly, it can be concluded that clinical phenotypes occur only when the STT3B level dramatically 
decreases with the mutation in both alleles of the gene.

In this study, we present a patient diagnosed with CDG-Ix with a novel homozygous mutation in STT3B and a rare clinical presentation of recurrent apnea and respiratory failure. Respiratory failure has rarely been reported in patients with CDG-Ix. In the present case, there was recurrent apnea and respiratory failure, which necessitated tracheostomy.

Although curative treatment cannot be initiated in most cases, definitive diagnosis is necessary for palliative care. Defining the disorder type is crucial in diagnosing the disorder, instructing parents, and offering suitable genetic counseling. ${ }^{6}$

\section{REFERENCES}

1. Freeze HH, Chong JX, Bamshad MJ, Ng BG. Solving glycosylation disorders: fundamental approaches reveal complicated pathways. Am J Hum Genet 2014; 94: 161-175.

2. Varki A. New and updated glycoscience-related resources at NCBI. Glycobiology 2017; 27: 993.

3. Fang J, Peters V, Assmann B, Körner C, Hoffmann GF. Improvement of CDG diagnosis by combined examination of several glycoproteins. J Inherit Metab Dis 2004; 27: 581-590.

4. Jaeken J, Hennet T, Matthijs G, Freeze HH. CDG nomenclature: time for a change! Biochim Biophys Acta 2009; 1792: 825-826.

5. McKenzie FA, Fietz M, Fletcher J, Smith RLL, Wright IMR, Jaeken J. A previously undescribed form of congenital disorder of glycosylation with variable presentation in siblings: early fetal loss with hydrops fetalis, and infant death with hypoproteinemia. Am J Med Genet A 2007; 143A: 2029-2034.

6. Morava E, Wosik H, Kártezi J, et al. Congenital disorder of glycosylation Type Ix: review of clinical spectrum and diagnostic steps. J Inherit Metab Dis 2008; 31: 450-456.

7. Lefeber DJ, Schönberg J, Morava E, et al. Deficiency of Dol-P-Man synthase subunit DPM3 bridges the congenital disorders of glycosylation with the dystroglycanopathies. Am J Hum Genet 2009; 85: 76-86.
8. Sinha MD, Horsfield C, Komaromy D, Booth CJ, Champion MP. Congenital disorders of glycosylation: a rare cause of nephrotic syndrome. Nephrol Dial Transplant 2009; 24: 2591-2594.

9. Jaeken J, Hennet T, Freeze HH, Matthijs G. On the nomenclature of congenital disorders of glycosylation (CDG). J Inherit Metab Dis 2008; 31: 669-672.

10. Jaeken J. Congenital disorders of glycosilation (CDG): it's all in it! J Inherit Metab Dis 2003; 26: 99118.

11. Dupré T, Vuillaumier-Barrot $S$, Chantret I, et al. Guanosine diphosphate-mannose: GlcNAc2-PPdolichol mannosyltransferase deficiency (congenital disorders of glycosylation type Ik): five new patients and seven novel mutations. J Med Genet 2010; 47: 729-735.

12. Jiang L, Zhu X, Chen J, Yang D, Zhou C, Hong Z. Two conserved oligosaccharyltransferase catalytic subunits required for $\mathrm{N}$-glycosylation exist in Spartina alterniflora. Bot Stud 2015; 56: 31.

13. Kitajima T, Xue W, Liu YS, et al. Construction of green fluorescence protein mutant to monitor STT3B-dependent N-glycosylation. FEBS J 2018; 285 : 915-928.

14. Sato T, Sako $\mathrm{Y}$, Sho $\mathrm{M}$, et al. STT3B-dependent posttranslational $\mathrm{N}$-glycosylation as a surveillance system for secretory protein. Mol Cell 2012; 47: 99110.

15. Moriconi C, Ordonez A, Lupo G, et al. Interactions between $\mathrm{N}$-linked glycosylation and polymerisation of neuroserpin within the endoplasmic reticulum. FEBS J 2015; 282: 4565-4579.

16. Shrimal S, Trueman SF, Gilmore R. Extreme C-terminal sites are posttranslocationally glycosylated by the STT3B isoform of the OST. J Cell Biol 2013; 201: 81-95.

17. Shrimal S, Ng BG, Losfeld ME, Gilmore R, Freeze $\mathrm{HH}$. Mutations in STT3A and STT3B cause two congenital disorders of glycosylation. Hum Mol Genet 2013; 22: 4638-4645.

18. Varki A. Biological roles of oligosaccharides: all of the theories are correct. Glycobiology 1993; 3: 97-130.

19. Rymen D, Jaeken J. Skin manifestations in CDG. J Inherit Metab Dis 2014; 37: 699-708.

20. Sparks SE, Krasnewich DM. Congenital Disorders of N-Linked Glycosylation and Multiple Pathway Overview. In: GeneReviews ${ }^{\circledR}$ [Internet]. University of Washington, Seattle; 2005. 